\title{
SYMPLECTIC REFLECTION ALGEBRAS IN POSITIVE CHARACTERISTIC
}

\author{
K.A. BROWN AND K. CHANGTONG
}

\begin{abstract}
Basic properties of symplectic reflection algebras over an algebraically closed field $k$ of positive characteristic are laid out. These algebras are always finite modules over their centres, in contrast to the situation in characteristic 0. For the subclass of rational Cherednik algebras, we determine the PI-degree and the Goldie rank, and show that the Azumaya and smooth loci of the centre coincide.
\end{abstract}

\section{INTRODUCTION}

1.1. Throughout this paper, $k$ will be an algebraically closed field of characteristic $p$, where $p$ is an odd prime. Symplectic reflection algebras over $\mathbb{C}$ were introduced in [12]. The same definition makes sense over $k$, and indeed symplectic reflection algebras over $k$ have been studied in [3] and [17. Let $(V, \omega)$ be a finite dimensional symplectic vector space over $k$ and $S(V)$ its symmetric algebra, and let $\Gamma$ be a finite subgroup of $\operatorname{Sp}(V)$ with chark $\nmid|\Gamma|$. The symplectic reflection algebra $H=H_{t, \mathbf{c}}(V, \omega, \Gamma)$ is a deformation of the skew group algebra $S(V) * \Gamma$; the precise definition is in (2.1). One can limit the study at once to the case where $\Gamma$ is generated by its set $S$ of symplectic reflections: that is, $s \in S$ if and only if $\operatorname{dim}_{k} \operatorname{im}(\operatorname{Id}-s)=2$. In the definition, $t \in k$ and $\mathbf{c}: S \longrightarrow k$ is a $\Gamma$-invariant function; $S(V) * \Gamma$ [resp. $\mathcal{D}(V) * \Gamma]$ corresponds to the case where $\mathbf{c}$ is the zero map and $t=0$ [resp. $t=1$ ].

1.2. Recall that Weyl algebras in positive characteristic are finite modules over their centres; see Lemma 5.1 In a parallel fashion, all symplectic reflection algebras over $k$ are finite modules over their centres by a result of Etingof [3, Appendix 10]. In contrast, over $\mathbb{C}$, when $t \neq 0, Z\left(H_{t, \mathbf{c}}\right)=\mathbb{C}$, 7, Proposition 7.2(2)]. When $t=0$ the theory over $k$ appears to be essentially the same as over $\mathbb{C}$. Thus the focus here will be on the case where $t$ is non-zero; in fact, after re-scaling, we may then assume that $t=1$.

Key words and phrases. symplectic reflection algebra; rational Cherednik algebra; fields of positive characteristic.

Part of the work described here was obtained in the $\mathrm{PhD}$ thesis of the second author, for which she was awarded a PhD by the University of Glasgow in 2006. Her PhD research was supported by a grant from the Thai Royal Government. We thank Iain Gordon and Catharina Stroppel for helpful comments. 
1.3. In $₫ 2$ minor adjustments to the characteristic 0 arguments suffice to show that $H=H_{1, \mathbf{c}}$ is a prime noetherian $k$-algebra with excellent homological properties namely, it is Auslander-regular and Cohen-Macaulay.

Let $e \in k \Gamma \subseteq H$ be the symmetrising idempotent $\frac{1}{|\Gamma|} \sum_{\gamma \in \Gamma} \gamma$. We show in $\$ 3$ that the symmetrising algebra $\mathrm{eHe}$ is a noetherian domain, a maximal order with - again - good homological properties. Just as in characteristic 0, the Satake homomorphism defines an isomorphism between $Z(H)$ and $Z(e H e)$. The algebras $H$ and $e H e$ are connected by a Morita context, whose details are laid out in (3.2) and (3.4).

1.4. In $\S \$$ and 5 we specialise to the case where $H$ is a rational Cherednik algebra, and prove our main results. That is, $\Gamma$ acts on a vector space $\mathfrak{h}$ of dimension $n$, with $\Gamma$ generated by pseudo-reflections for this action, and $V=\mathfrak{h} \oplus \mathfrak{h}^{*}$ with the canonical $\Gamma$-invariant symplectic form, denoted by $\omega$.

Theorem. Let $k, \mathfrak{h}, n, \Gamma, \omega$ and $H$ be as above. Denote the centre of $H$ by $Z(H)$.

(i) $H$ is a free module of rank $p^{2 n}|\Gamma|^{3}$ over the central subalgebra

$$
Z_{0}:=\left(S(\mathfrak{h})^{\mathrm{p}}\right)^{\Gamma} \otimes\left(S\left(\mathfrak{h}^{*}\right)^{\mathrm{p}}\right)^{\Gamma},
$$

where $(-)^{\mathrm{p}}$ denotes the Frobenius map.

(ii) The Goldie rank of $H$ is $|\Gamma|$.

(iii) The PI-degree of $H$ is $p^{n}|\Gamma|$.

(iv) Every irreducible $H$-module of maximal $k$-dimension $p^{n}|\Gamma|$ is a regular $k \Gamma$ module of rank $p^{n}$.

(v) The Azumaya locus of $H$ is equal to the smooth locus of $\operatorname{Maxspec}(Z(H))$ : that is, for a maximal ideal $\mathfrak{m}$ of $Z(H), \mathfrak{m}$ annihilates a simple $H$-module of the maximal possible $k$-dimension $p^{n}|\Gamma|$ if and only if $Z(H)_{\mathfrak{m}}$ has finite global dimension.

1.5. In the last section, $\sqrt{6}$, we gather together some questions and conjectures arising from this work.

\section{DEFINITIONS AND BACKGROUND}

2.1. Recall the hypothesis on $k$ from 1.1. When we want to emphasise that a particular result is valid over all algebraically closed fields $K$ such that

$$
2|\Gamma| \text { is a unit in } K
$$

,we will always denote the ground field by $K$. All fields will be assumed to satisfy (11). The ingredients needed for the construction of a symplectic reflection algebra are a finite dimensional symplectic $K$-vector space $(V, \omega)$ and a finite group $\Gamma$ of symplectic automorphisms of $V$. An element $\gamma \in \Gamma$ is called a symplectic reflection (on $V$ ) if the rank of Id $-\gamma$ is 2 . Let $S$ denote the set of symplectic reflections in $\Gamma$ and let $\mathbf{c}: S \longrightarrow K: s \mapsto c_{s}$ be a $\Gamma$-invariant map. Let $t \in K$. The symplectic 
reflection algebra $H_{t, \mathbf{c}}$ [12, page 245] is the deformation of the skew group algebra $S(V) * \Gamma$ obtained by replacing the commutativity relations $x y-y x=0$ defining the polynomial algebra $S(V)$ by new relations

$$
x y-y x=t \omega(x, y) 1_{\Gamma}-\sum_{s \in S} c_{s} \omega_{s}(x, y) s,
$$

for all $x, y \in V$. Here, $w_{s}$ is the skew-symmetric form on $V$ which has $\operatorname{ker}(\operatorname{Id}-s)$ in its radical, and coincides with $\omega$ on $\operatorname{im}(\operatorname{Id}-s)$. Thus, writing $T(V)$ for the tensor algebra of $V, H_{t, \mathbf{c}}$ is the factor of the skew group algebra $T(V) * \Gamma$ by the ideal generated by the elements (2). Throughout this paper we will denote the dimension of $V$ by $2 n$, and we'll assume for convenience that $\Gamma=\langle S\rangle$.

2.2. PBW theorem and homological consequences. It's clear from the definition that if $H_{t, \mathbf{c}}=H_{t, \mathbf{c}}(V, \omega, \Gamma)$ is a symplectic reflection algebra, then $H_{t, \mathbf{c}}$ is an $\mathbb{N}$-filtered algebra with filtration $\mathcal{F}_{0}:=K \Gamma, \mathcal{F}_{1}:=K \Gamma+K \Gamma V$, and $\mathcal{F}_{i}:=\mathcal{F}_{1}^{i}$ for $i \geq 1$. It's also immediate from the relations (2) that there is an algebra epimorphism

$$
\pi: S(V) * \Gamma \longrightarrow \operatorname{gr}_{\mathcal{F}}\left(H_{t, \mathbf{c}}\right)
$$

the starting point for the study of symplectic reflection algebras is 12 , Theorem 1.3], the "Poincaré-Birkhoff-Witt theorem", which asserts that

$$
\pi \text { is an isomorphism. }
$$

Equivalently, the identity map on $V$ and $\Gamma$ extends to a vector space isomorphism between $H_{t, \mathbf{c}}$ and $S(V) \otimes \Gamma$. Some of the consequences flowing from this fact are gathered in the theorem below, for which we need to recall a few definitions. A noetherian algebra $A$ is Auslander-Gorenstein if $A$ has finite left injective dimension $d$, and for every finitely generated left $A$-module $M$, every non-negative integer $i$ and every submodule $N$ of $\operatorname{Ext}_{A}^{i}(M, A)$, we have $\operatorname{Ext}_{A}^{j}(N, A)=0$ for all $j<i$; and the same conditions hold with left replaced by right throughout. By a theorem of Zaks [28, if a noetherian algebra has finite right and left injective dimensions, then these are equal. If $A$ is Auslander-Gorenstein and has finite global dimension, then $A$ is called Auslander-regular, (and then necessarily gldim $(A)=d$ ). The grade $j_{A}(M) \in \mathbb{N} \cup\{+\infty\}$ of a non-zero finitely generated $A$-module $M$ is the least integer $j$ such that $\operatorname{Ext}_{A}^{j}(M, A)$ is non-zero. If $A$ has finite Gel'fand-Kirillov dimension, then $A$ is Cohen-Macaulay if

$$
j_{A}(M)+\mathrm{GK}-\operatorname{dim}(M)=\mathrm{GK}-\operatorname{dim}(A)
$$

for every non-zero finitely generated left or right $A$-module $M$. We recall that if $A$ has a positive filtration such that $\operatorname{gr}(A)$ is Auslander-Gorenstein, and $M$ is a finitely generated $A$-module endowed with a standard filtration, then

$$
j_{A}(M)=j_{\operatorname{gr}(A)}(\operatorname{gr}(M))
$$


by [4. (1) in the proof of Theorem 3.9]; so that, since

$$
\mathrm{GK}-\operatorname{dim}(M)=\mathrm{GK}-\operatorname{dim}_{\operatorname{gr}(A)}(\operatorname{gr}(M))
$$

by [22, Proposition 8.6.5],

$$
\text { if } \operatorname{gr}(A) \text { is Cohen-Macaulay, then so also is } A \text {. }
$$

The following basic facts are well known, and were for the most part proved for algebras over $\mathbb{C}$ in [12]; the proofs over a general field $k$ are identical, depending on filtered graded techniques and the corresponding statements for $S(V) * \Gamma$, once one knows from (3) that $\operatorname{gr}_{\mathcal{F}}\left(H_{t, \mathbf{c}}\right) \cong S(V) * \Gamma$.

Theorem. Let $K$ be an arbitrary field, and let $H_{t, \mathbf{c}}=H_{t, \mathbf{c}}(V, \omega, \Gamma)$ be a symplectic reflection algebra, with $\operatorname{dim}_{K}(V)=2 n$. Then the following hold.

(i) $H_{t, \mathbf{c}}$ is a prime noetherian algebra.

(ii) $H_{t, \mathbf{c}}$ has finite Gel'fand-Kirillov dimension, $\mathrm{GK}-\operatorname{dim}\left(H_{t, \mathbf{c}}\right)=2 n$.

(iii) $H_{t, \mathbf{c}}$ is Auslander-regular and Cohen-Macaulay, with $\operatorname{gldim}\left(H_{t, \mathbf{c}}\right) \leq 2 n$.

Proof. (i) [22, Theorem 1.6.9] and [9, Ex.6.6].

(ii) [22, Proposition 8.6.5] and [16, Proposition 5.5].

(iii) First, $S(V) * \Gamma$ is Auslander-regular by [27. This property lifts to $H_{t, \mathbf{c}}$ by 4. Theorem 3.9 and the Remark following]. The Cohen-Macaulay property is dealt with in the discussion preceding the theorem.

2.3. The centre. There is a fundamental dichotomy in the theory determined by whether or not the parameter $t$ is zero. Since $H_{t \mathbf{c}} \cong H_{\lambda t, \lambda \mathbf{c}}$ for $0 \neq \lambda \in k$, we need consider only the cases $t=0$ and $t=1$.

Theorem. Let $H_{t, \mathbf{c}}=H_{t, \mathbf{c}}(V, \omega, \Gamma)$ be a symplectic reflection algebra over an arbitrary field $K$.

(i) (12, Theorem 3.1], [7, Proposition 7.2(2)]) Suppose that $K$ has characteristic 0 . If $t=0$, then $H_{0, \mathbf{c}}$ is a finite module over its centre $Z\left(H_{0, \mathbf{c}}\right)$, which is Gorenstein. If $t=1$, then $Z\left(H_{1, \mathbf{c}}\right)=K$.

(ii) Suppose for the rest of the theorem that $K=k$.

(a) (Etingof, [3, Appendix 10]) $H_{t, \mathbf{c}}$ is a finite module over its centre for all values of $t$. With respect to the filtration $\left\{F_{i}\right\}$ of (2.2), the associated graded algebra of $Z=Z\left(H_{1, \mathbf{c}}\right)$ is $\left(S(V)^{\mathrm{p}}\right)^{\Gamma}$, where $(-)^{\mathrm{p}}$ denotes the Frobenius homomorphism.

(b) $Z\left(H_{t, \mathbf{c}}\right)$ is Gorenstein.

Proof. The statements for $t=0$ are given by [12, Theorem 3.3], for all ground fields. The only other claim which is not as stated in the cited references is (ii)(b) for non-zero $t$. For this, by [4, Theorem 3.9] it's enough to prove that $\operatorname{gr}(Z(H))$ is Gorenstein. By the second statement in (ii), bearing in mind that $\left(S(V)^{\mathrm{p}}\right)^{\Gamma}=$ 
$\left(S(V)^{\Gamma}\right)^{\mathrm{p}}, \operatorname{gr}(Z(H))$ is isomorphic to $S(V)^{\Gamma}$. Since $\Gamma \subseteq S L(V), S(V)^{\Gamma}$ is Gorenstein by Watanabe's theorem [26.

It should be noted that the uncertainty over the precise value of the global dimension of $H_{t, \mathbf{c}}$ noted in Theorem 2.2(iii) disappears when its centre is big. For in this case every irreducible $H_{t, \mathbf{c}}-$ module $M$ is a finite dimensional $K$-vector space, so that GK $-\operatorname{dim}(M)=0$. Therefore, by (4) and Theorem 2.2(ii), pr.dim $(M)=2 n$, and so we deduce the

Theorem. Let $K$ be an arbitrary field satisfying (1), and suppose that the symplectic reflection algebra $H=H_{t, \mathbf{c}}(V, \omega, \Gamma)$ is a finite module over its centre. Then

$$
\operatorname{gldim}(H)=\text { Krull } \operatorname{dim}(H)=\mathrm{GK}-\operatorname{dim}(H)=\operatorname{dim}(V) .
$$

\section{INTERPLAY WITH THE SPHERICAL SUBALGEBRA}

For the rest of the paper our ground field will be $k$, and we focus on the case where $k$ has positive characteristic and the theory deviates from that over characteristic 0 ; that is, we shall assume that the parameter $t$ is non-zero, so that, after re-scaling, we can assume that $t=1$. So from now on $H$ will denote a symplectic reflection $k$-algebra $H:=H_{1, \mathbf{c}}(V, \omega, \Gamma)$.

3.1. Basic properties. Recall that, following [12, the symmetrising idempotent $e$ of $H$ is $e=\frac{1}{|\Gamma|} \sum_{\gamma \in \Gamma} \gamma$, and $e H e$ is the spherical subalgebra of $H$. (Note that it is not a subalgebra of $H$ in the usual sense of the word, since it doesn't contain $1_{H}$.) The filtration $\left\{\mathcal{F}_{i}\right\}$ of $H$ induces a filtration $\{e \mathcal{F} e\}$ of $e H e$ with $i^{\text {th }}$ subspace $\mathcal{F}_{i} H \cap e H e=e \mathcal{F}_{i} H e$. It follows that

$$
\operatorname{gr}_{e \mathcal{F} e}(e H e)=e \operatorname{gr}_{\mathcal{F}}(H) e=e S(V) * \Gamma e \cong S(V)^{\Gamma} .
$$

Standard filtered-graded methods can therefore be used to deduce the following theorem. For (v), we recall the definition of a maximal order. Suppose that the noetherian algebra $A$ is prime, so that it has a simple artinian quotient ring $Q$ by Goldie's theorem, [22, Theorem 2.3.6]. There is an equivalence relation on orders in $Q$ defined by: $R \sim S$ if and only if there exist units $a, b, c, d$ of $Q$ with $a R b \subseteq S$ and $c S d \subseteq R, 22,5.1 .1,3.1 .9]$. Then $A$ is a maximal order if it is a maximal member of its equivalence class of orders in $Q$. A commutative noetherian integral domain is a maximal order if and only if it is integrally closed, [22, Lemma 5.3.2].

Theorem. (i) eHe is a finitely generated module over its centre eZe.

(ii) eHe is an affine noetherian domain.

(iii) $\mathrm{He}$ is a finitely generated right eHe-module.

(iv) eHe is Auslander-Gorenstein and Cohen-Macaulay.

(v) eHe is a maximal order. 
Proof. (i) is clear from Theorem 2.3(ii)(a). Note next that $S(V)^{\Gamma}$ is affine and $S(V)$ is a finitely generated $S(V)^{\Gamma}$-module by the Hilbert-Noether theorem, 2, Theorem 1.3.1]; thus $e H e$ is affine by (6), and since $\operatorname{gr}(H e) \cong S(V)$ (iii) follows also. The rest of (ii) follows from (6) and [22, Theorems 1.6.9] and [9, Ex. 6.6].

To prove (iv) we first observe that $S(V)^{\Gamma}$ is Gorenstein by Watanabe's theorem 26. Theorem 1], since $\Gamma \subseteq \operatorname{Sp}(V) \subseteq S L(V)$. The Auslander-Gorenstein condition thus follows from [18, Chapter II, Proposition 2.2.1]. Moreover, since $S(V)^{\Gamma}$ is Cohen-Macaulay, either by the Hochster-Eagon theorem [2, Theorem 4.3.6], or since commutative Gorenstein rings are Cohen-Macaulay, it follows from (5) in $\$ 2.2$ that $e H e$ is Cohen-Macaulay. For (v) it's enough, in view of [22, Theorem 5.1.6] and (6), to note that the fixed ring $S(V)^{\Gamma}$ is integrally closed, [2, Proposition 1.1.1].

3.2. The Morita context. In this paragraph we prove a version of [12, Theorem 1.5] for symplectic reflection algebras of positive characteristic. For the most part the proofs follow the proof of [12, Theorem 1.5], so we only indicate the key steps. Part (ii) is an example of a double centralizer property in the spirit of [15].

Theorem. (i) $\operatorname{End}_{H}(H e) \cong e H e$.

(ii) $\operatorname{End}_{e H e}(H e) \cong H$.

(iii) $\operatorname{Hom}_{e H e}\left(H_{\mid}, e H e_{\mid}\right) \cong e H$, and $\operatorname{Hom}_{e H e}\left(\left.\right|_{\mid} e H, \mid e H e\right) \cong H e$. In particular, $\mathrm{He}$ is a reflexive right $\mathrm{eHe}-$ module, and $\mathrm{eH}$ is left $\mathrm{eHe}-$ reflexive.

Proof. (Sketch) Of course (i) is clear.

(ii) Step 1: Construction of an ascending filtration on $\operatorname{End}_{e H e}(H e)$. Since $H e$ is filtered by $\left\{\mathcal{F}_{i} H e\right\}$, we can choose a finite set of generators $u_{1}, \ldots, u_{l}$ of $\operatorname{gr}_{\mathcal{F}}(H e)$, whose lifts $\left\{\tilde{u}_{1}, \ldots, \tilde{u}_{l}\right\}$ generate the $e H e$-module $H e$. Let $\tilde{u}_{i} \in \mathcal{F}_{d_{i}}(H e)$. We can assume that $\mathcal{F}_{n}(H e)=\sum_{j=1}^{l} \tilde{u}_{j} \mathcal{F}_{n-d_{j}}(e H e)$ for all $n>>0$. Hence, for $f \in \operatorname{End}_{e H e}(H e)$, there is a non-negative integer $m$ such that $f\left(\mathcal{F}_{n} H e \subseteq \mathcal{F}_{n+m} H e\right.$ for all $n \geq 0$. In this case we say that $f \in \mathcal{F}_{m}$ End; in this way $\operatorname{End}_{e H e}(H e)$ acquires an ascending filtration.

Step 2: The map $\eta: H \longrightarrow \operatorname{End}_{e H e}(H e)$. For $h \in H$, let $\eta(h)$ denote left multiplication of $\mathrm{He}$ by $h$, so it's clear that $\eta: H \longrightarrow \operatorname{End}_{e H e}(H e)$ is an algebra homomorphism. We have to prove that $\eta$ is an isomorphism. Since $\eta$ preserves the filtrations it induces a homomorphism $\operatorname{gr}(\eta)$ of the corresponding graded algebras, and it's enough to prove that the latter is an isomorphism. Consider then the composition

$$
\begin{aligned}
& S(V) * \Gamma \cong \operatorname{gr}(H) \stackrel{\operatorname{gr}(\eta)}{\longrightarrow} \operatorname{gr}\left(\operatorname{End}_{e H e}(H e)\right) \\
& \stackrel{j}{\longrightarrow} \operatorname{End}_{\operatorname{gr}(e H e)}(\operatorname{gr}(H e)) \cong \operatorname{End}_{S(V)^{\Gamma}}(S(V)),
\end{aligned}
$$

where $j$ is the obvious homomorphism. Injectivity follows because, after tensoring with the quotient field $Q\left(S(V)^{\Gamma}\right)$ of $\left.S(V)^{\Gamma}\right)$, the induced map $\psi:=\operatorname{Id} \otimes_{S(V)^{\Gamma}}(j \circ$ $\operatorname{gr}(\eta))$ is easily seen to be injective. 
Step 3: Surjectivity of $\eta$. We prove this by demonstrating surjectivity of $j \circ g r(\eta)$, and claim first that

$$
\psi \text { is surjective. }
$$

This follows because $\psi$ is an injective homomorphism from $Q(S(V)) * \Gamma$ to $\operatorname{End}_{Q\left(S(V)^{\Gamma}\right)}(Q(S(V)))$, and these two $Q\left(S(V)^{\Gamma}\right)$-vector spaces have the same dimension, namely $|\Gamma|^{2}$. Hence $j \operatorname{ogr}(\eta)(S(V) * \Gamma) \subseteq \operatorname{End}_{S(V)^{\Gamma}}(S(V))$, and these two algebras have the same simple artinian quotient ring, namely $\operatorname{End}_{Q\left(S(V)^{\Gamma}\right)}(Q(S(V)))$. But note that, since $S(V)$ is a finitely generated $S(V)^{\Gamma}$-module, so too is $\operatorname{End}_{S(V)^{\Gamma}}(S(V))$.

A fortiori, $\operatorname{End}_{S(V)^{\Gamma}}(S(V))$ is a finitely generated module over its subalgebra $j \circ \operatorname{gr}(\eta)(S(V) * \Gamma)$. However this last algebra is a maximal order, by 19, Theorem 4.6], so the inclusion of algebras must be an equality as required.

(iii) This follows from (ii) just as in [12] - that is, one confirms using (ii) that the map from $e H$ to $\operatorname{Hom}_{e H e}(H e, e H e)$ induced by left multiplication is an isomorphism of right $e \mathrm{He}-$ modules. The second statement follows symmetrically.

Corollary A. $H$ is a maximal order.

Proof. Suppose that $T$ is an order in $Q(H)$ with $H \subseteq T$ and $T$ equivalent to $H$. By [22, Lemma 3.1.10], there is a non-zero ideal $I$ of $H$ with either $I T \subseteq H$ or $T I \subseteq H$. Suppose the former holds. Then $0 \neq e I e \triangleleft e H e$, since $H$ is prime, with

$$
(e I e)(e T e) \subseteq e I T e \subseteq e H e .
$$

Thus $e T e$ is an order in $e Q(H) e=Q(e H e)$, which contains $e H e$ and is equivalent to it. So, by Theorem 3.1(v),

$$
e T e=e H e .
$$

Note that, if instead $T I \subseteq H$, we can still arrive at (8). Bearing in mind the identifications of Theorem 3.2(iii), (8) shows that $T e \subseteq(e H)^{*}$, and so, by Theorem 3.2(iii),

$$
T e=H e .
$$

In other words, $T \subseteq \operatorname{End}_{e H e}(H e)$. From Theorem 3.2 (ii) we deduce that $T=H$, as required.

Remarks. (i)The proof of the corollary works over fields of characteristic 0 , and is independent of the value of the parameter $t$; in these other cases the result does not seem to have been previously recorded.

(ii) One would naturally expect to prove the above corollary by lifting the result using filtered-graded methods from the corresponding result for the skew group algebra $S(V) * \Gamma \cong \operatorname{gr}_{F}(H)$. However the relevant lifting theorem in the literature, 
Chamarie's theorem [22, Theorem 5.1.6], requires the algebras involved to be domains. While this defect can presumably be rectified, it seems more efficient to proceed as above.

Below and in (3.4) we need the concept of a localizable prime ideal $P$ of a noetherian ring $R$ : this means that the set $\mathcal{C}(P)$ of elements of $R$ whose images modulo $P$ are not zero divisors forms a (right and left) Ore set in $R$. When this happens, one can invert the elements of $\mathcal{C}(P)$ to obtain the local ring $R_{P}$, a partial quotient ring of the factor ring of $R$ by the ideal $I=\{r \in R:$ cr $=0$ or $r c=$ 0 for some $c \in \mathcal{C}(P)\}$. When $R$ is a finite module over its centre $Z$, a prime ideal $P$ of $R$ is localizable when (and, in fact, only when) it is the unique prime of $R$ lying over $P \cap Z$, and one sees easily that in this case we can form $R_{P}$ by inverting the elements of $Z \backslash(P \cap Z)$. For background on these ideas, see, for example, [13].

Standard Morita theory [22, Proposition 3.5.6] applied to the theorem above tells us that $H$ is Morita equivalent to $e H e$ exactly when $\mathrm{HeH}=H$. More precisely, the size of $\mathrm{HeH}$ indicates how close $\mathrm{H}$ and $e \mathrm{He}$ are to being Morita equivalent. In this connection, we thus have:

Lemma. Let $e$ be the symmetrising idempotent of $H$. Let $\mathfrak{p}$ be a prime ideal of $Z:=Z(H)$ with $H e H \cap Z \subseteq \mathfrak{p}$. Then $\mathfrak{p}$ has height at least 2 .

Proof. Suppose the result is false: so there is a prime $\mathfrak{p}$ of $Z$ of height 1 , with

$$
H e H \cap Z \subseteq \mathfrak{p} .
$$

Thus, we can localize at $\mathfrak{p}$ by inverting the elements $Z \backslash \mathfrak{p}$, to obtain the ring $H_{\mathfrak{p}}$. Since $H$ is a prime maximal order and is a finite module over its centre, by Theorems 2.2(i) and 2.3(ii), and Corollary 3.2A, all its height one primes are localizable, by 21. Propositions II.2.2 and II.2.6, and Theorème IV.2.15]. Equivalently, by [5, Theorem III.9.2] or [23, Theorem 7], there is a unique height one prime of $H$ lying over $\mathfrak{p}$ - let's call it $P$. Thus $H_{\mathfrak{p}}$ is a local ring with Jacobson radical $P H_{\mathfrak{p}}$. Now (9) ensures that $H_{\mathfrak{p}} e H_{\mathfrak{p}}$ is a proper ideal of $H_{\mathfrak{p}}$, so that $e \in P H_{\mathfrak{p}}$, the Jacobson radical of $H_{\mathfrak{p}}$. Hence $1-e$ is a unit in $H_{\mathfrak{p}}$, a contradiction. So the result is proved.

Corollary B. Let $\mathfrak{p}$ be any prime ideal of $Z$ such that $H e H \cap Z$ is not contained in $\mathfrak{p}$. Then $H_{\mathfrak{p}}$ is Morita equivalent to $e H_{\mathfrak{p}} e$. In particular, this is true for every prime ideal of $Z$ of height 1 .

3.3. The Satake homomorphism. The proof of the next result also follows the corresponding argument used to prove [12, Theorem 3.1]; but note the important difference that, when $t=0, e H e$ is commutative.

Theorem. The map $\theta: H \rightarrow e H e: u \mapsto$ eue is an algebra homomorphism when restricted to the centre $Z$ of $H$, and maps $Z$ isomorphically to the centre of eHe. 
Proof. (Sketch) It's obvious that the restriction of $\theta$ to $Z$ is an algebra homomorphism. We define an inverse map $\xi: Z(e \mathrm{He}) \longrightarrow Z$ as follows. Let eae $\in Z(e \mathrm{He})$, so that right multiplication of $\mathrm{He}$ by eae defines a right $e \mathrm{He}$-module endomorphism $r_{e a e}$ of $H e$. By Theorem 3.2(ii) this endomorphism must be induced by left multiplication of $H e$ by an element $\xi(e a e)$ of $H$; moreover, since $r_{e a e}$ commutes with the left multiplications of $H e$ by the elements of $H, \xi(e a e) \in Z$. It's now clear that $\xi$ is an algebra homomorphism, and easy to check that it is inverse to $\theta_{\mid Z}$.

3.4. The Morita context revisited. An important theme in the study of those symplectic reflection algebras $H$ which are finite modules over their centres $Z$ has been the determination of the groups and parameter values (if any) for which the centre is smooth [12, 14, 20, [1.

Recall [5, Theorem III.1.6] that a prime $k$-algebra $A$, finitely generated as a module over over its affine centre $Z$, is Azumaya over $Z$ if (and only if) all the irreducible $A$-modules have the same vector space dimension (which is then necessarily equal to the PI-degree of $A$. As we'll see in (5.3), the smoothness of $Z(H)$ is closely related to the question of when $H$ is Azumaya over $Z$. Regarding this latter question, we have

Theorem. Let $P$ be a prime ideal of $H$, and let $\mathfrak{p}$ be the prime $P \cap Z$ of $Z:=Z(H)$. Consider the following statements:

(i) $\mathfrak{p}$ is in the Azumaya locus.

(ii) There exists a positive integer s such that

$$
H_{\mathfrak{p}} \cong M_{s}\left(e H_{\mathfrak{p}} e\right)
$$

and $e H_{\mathfrak{p}} e$ is a local ring with Jacobson radical $e \mathfrak{p} H_{\mathfrak{p}} e$.

(iii) $P$ is a localizable ideal of $H$.

(iv) $P$ is the unique prime ideal of $H$ lying over $\mathfrak{p}$.

(v) $H e H \cap Z \nsubseteq \mathfrak{p}$, and ePe is the unique prime ideal of eHe lying over epe.

(vi) There exists a positive integer s such that

$$
H_{\mathfrak{p}} \cong M_{s}\left(e H_{\mathfrak{p}} e\right)
$$

and $e H_{\mathfrak{p}}$ e is a local ring.

Then (i) $\Leftrightarrow($ ii) $\Rightarrow$ (iii) $\Leftrightarrow($ iv) $\Leftrightarrow(v) \Leftrightarrow(v i)$.

Proof. $(i) \Rightarrow($ iii $)$ : This is clear, since, if $\mathfrak{p}$ is Azumaya in $H$, then inverting $Z \backslash \mathfrak{p}$ yields the local ring $H_{P}$.

$($ iii $) \Leftrightarrow(i v)$ : This is Müller's theorem, [23, Theorem 7] or [5, Theorem III.9.2].

$(i v) \Rightarrow(v)$ : Assume (iv), which by the above is equivalent to (iii). If $H e H \cap Z \subseteq$ $\mathfrak{p}$, then $H_{P} e H_{P}$ is a proper ideal of $H_{P}=H[Z \backslash \mathfrak{p}]^{-1}$, and this is impossible just as in the proof of Lemma 3.2. This proves the first part of (v); the second part follows from Corollary 3.2, which shows that $e H_{P} e=e H_{\mathfrak{p}} e$ is local with Jacobson radical $e P H_{P} e$. 
$(v) \Rightarrow(v i)$ : Assume (v). By Corollary $3.2 H_{\mathfrak{p}}$ is Morita equivalent to $e H_{\mathfrak{p}} e$, and the latter ring is local by $(\mathrm{v})$. Now $H_{\mathfrak{p}} e$ is a finitely generated projective right module over the local domain $e H_{\mathfrak{p}} e$, by the Morita theorems and Theorem 2.2(ii) and (iii). Hence $H_{\mathfrak{p}} e_{\mid e H_{\mathfrak{p}} e}$ is free of finite rank $s$, and its endomorphism ring $H_{\mathfrak{p}}$ is thus isomorphic to $M_{s}\left(e H_{\mathfrak{p}} e\right)$.

$(v i) \Rightarrow(i i i)$ : Assume (vi). Then $H_{\mathfrak{p}}$ is a local ring in which $P H_{\mathfrak{p}}$ is a maximal ideal. Thus $P H_{\mathfrak{p}}$ must be the Jacobson radical of $H_{\mathfrak{p}}$, so $P$ is localizable, proving (iii).

(ii) $\Rightarrow(i)$ : If (ii) holds, then $H_{\mathfrak{p}}$ is a local ring whose Jacobson radical is generated by its intersection with the centre. Thus $H_{\mathfrak{p}}$ is Azumaya, by [5, Theorem III.1.6].

$(i) \Rightarrow(i i)$ : Assume (i). Then, as we have seen, (vi) holds, and, writing $J(R)$ for that Jacobson radical of a ring $R$,

$$
J\left(H_{\mathfrak{p}}\right) \cong J\left(M_{s}\left(e H_{\mathfrak{p}} e\right)\right)=M_{s}\left(J\left(e H_{\mathfrak{p}} e\right)\right)=M_{s}\left(e \mathfrak{p} H_{\mathfrak{p}} e\right),
$$

since $\mathfrak{p}$ is Azumaya.

Remarks. (i) We expect that (i) - (vi) in the above theorem should be equivalent. This is true for all symplectic reflection algebras (over any field) at $t=0$, by [12, Theorem 1.7]. But the proof depends crucially on the commutativity of $e \mathrm{He}$, in particular [12, Lemma 2.24]. When $k$ has positive characteristic and $t=1$ we can't prove the equivalence of (i) - (vi) even in the case of Cherednik algebras; the difficulty lies in our inadequate understanding of the relation of the maximal order $e H e$ to its centre.

(ii) We expect the integer $s$ of Theorem[3.4(vi) to be $|\Gamma|$; we prove this in Theorem 4.4 when $H$ is a Cherednik algebra.

\section{Cherednik Algebras - Structure}

4.1. For the rest of the paper we shall assume that $H$ is a rational Cherednik algebra over an algebraically closed field $k$ of positive characteristic $p$. We fix a finite dimensional $k$-vector space $\mathfrak{h}$ and a finite group $\Gamma$ of automorphisms of $\mathfrak{h}$. We shall assume throughout that $\Gamma$ is generated by pseudo-reflections for its action on $\mathfrak{h}$. Then $\Gamma$ acts on $V:=\mathfrak{h} \oplus \mathfrak{h}^{*}$, and this space admits a canonical $\Gamma$-invariant symplectic form $\omega$, defined by $\omega((u, f),(x, g)):=g(u)-f(x)$ for $u, x \in \mathfrak{h}$ and $f, g \in \mathfrak{h}^{*}$. The set $S$ of pseudo-reflections in $\Gamma$ is then the set of symplectic reflections for $\Gamma$ acting on $V$, so - as in (2.1) - we can choose $t \in k$ and a $\Gamma$-invariant function c $: S \longrightarrow k$, and define the symplectic reflection algebra $H_{t, \mathbf{c}}:=H_{t, \mathbf{c}}(V, \omega, \Gamma)$. We shall continue to assume throughout that

$$
t=1 .
$$


4.2. The central invariant subalgebra. Let $H=H_{1, \mathbf{c}}:=H_{1, \mathbf{c}}\left(\mathfrak{h} \oplus \mathfrak{h}^{*}, \omega, \Gamma\right)$ be a rational Cherednik algebra. Notice that the skew group algebras $S(\mathfrak{h}) * \Gamma$ and $S\left(\mathfrak{h}^{*}\right) * \Gamma$ are contained in $H$, as is clear from the defining relations of $H$ and the Poincaré-Birkhoff-Witt theorem (3) . Hence the centres of these algebras, $S(\mathfrak{h})^{\Gamma}$ and $S\left(\mathfrak{h}^{*}\right)^{\Gamma}$, are also in $H$. Less trivially, it is proved in [12, Proposition 4.15] using Dunkl operators that, when $t=0$ and $k$ has characteristic 0 ,

$$
S(\mathfrak{h})^{\Gamma} \otimes S\left(\mathfrak{h}^{*}\right)^{\Gamma} \subseteq Z(H) ;
$$

an alternative proof of the same result is given in [14, Proposition 3.6]. When $t=1$ and the characteristic is positive, $H_{1,0}$ is $D(\mathfrak{h}) * \Gamma$, the skew group algebra of the Weyl algebra, so its centre is easily calculated to be $\left(S\left(\mathfrak{h} \oplus \mathfrak{h}^{*}\right)^{\mathrm{p}}\right)^{\Gamma}$. It's obvious, therefore, what the characteristic $p$ analogue of (10) should be; we offer here a proof of the result which is completely elementary and can be adapted to give also an easy proof of the original characteristic 0 theorem.

Proposition. Let $H=H_{1, \mathbf{c}}\left(\mathfrak{h} \oplus \mathfrak{h}^{*}, \omega, \Gamma\right)$ be a rational Cherednik algebra. Then

$$
Z_{0}:=\left(S(\mathfrak{h})^{\mathrm{p}}\right)^{\Gamma} \otimes\left(S\left(\mathfrak{h}^{*}\right)^{\mathrm{p}}\right)^{\Gamma} \subseteq Z(H) .
$$

Proof. Fix a pair of dual bases $\left\{x_{i}\right\}$ and $\left\{y_{i}\right\}$ of $\mathfrak{h}$ and $\mathfrak{h}^{*}$. Thus the filtration $\left\{\mathcal{F}_{i}\right\}$ of (2.2) is defined by setting degree $\left(x_{i}\right)=\operatorname{degree}\left(y_{i}\right)=1$ and degree $(\gamma)=0$, for $1 \leq i \leq n$ and $\gamma \in \Gamma$. Note that $H$ is also a $\mathbb{Z}$-graded algebra, with degree $\left(x_{i}\right)=1$, degree $\left(y_{i}\right)=-1$, and degree $(\gamma)=0$, for $1 \leq i \leq n$ and $\gamma \in \Gamma$. Let's denote the $i$ th graded subspace by $\mathcal{M}_{i}(H)$, so $H=\oplus_{i \in \mathbb{Z}} \mathcal{M}_{i}(H)$, and, putting $\mathcal{M}_{i}(Z):=$ $\mathcal{M}_{i}(H) \cap H$,

$$
Z:=Z(H)=\oplus_{i \in \mathbb{Z}} \mathcal{M}_{i}(Z) .
$$

Let $f$ be a homogeneous element of $\left(S(\mathfrak{h})^{\mathrm{p}}\right)^{\Gamma}$ of degree $m$. We aim first to show that

$$
f \in Z \text {. }
$$

Let $\sigma_{m}^{\mathcal{F}}: \mathcal{F}_{m} Z \longrightarrow \mathcal{F}_{m} Z / \mathcal{F}_{m-1} Z$ be the symbol map of degree $m$. Since $f \in S(\mathfrak{h})$, its $\mathcal{F}$-degree and $\mathcal{M}$-degree are the same, namely

$$
\operatorname{degree}_{\mathcal{F}}(f)=\operatorname{degree}_{\mathcal{M}}(f)=m .
$$

Note that $\left(S(V)^{\Gamma}\right)^{\mathrm{p}} \cong\left(S(V)^{\mathrm{p}}\right)^{\Gamma}$, by the $\Gamma$-equivariance of the Frobenius homomorphism. Thus, by Theorem 2.3(ii)(a), there exists $z \in \mathcal{F}_{m} Z$ with $\sigma_{m}^{\mathcal{F}}(z)=f$. On the other hand, by (11), we can write

$$
z=z_{1}+z_{2},
$$

where $z_{1} \in \mathcal{M}_{m}(Z)$ and $z_{2} \in \bigoplus_{j \neq m} \mathcal{M}_{j}(Z)$. Now, by the Poincaré-Birkhoff-Witt theorem, (2.2) (3), there is no cancellation between $\sigma_{m}^{\mathcal{F}}\left(z_{1}\right)$ and $\sigma_{m}^{\mathcal{F}}\left(z_{2}\right)$. That is,

$$
f=\sigma_{m}^{\mathcal{F}}(z)=\sigma_{m}^{\mathcal{F}}\left(z_{1}\right)+\sigma_{m}^{\mathcal{F}}\left(z_{2}\right) .
$$


Since $\mathcal{M}$-degree $(f)=m$,

$$
z_{1}=f+g
$$

where $g \in \mathcal{M}_{m}(H)$. We claim that $g=0$. Suppose $g \neq 0$. If there is a monomial $\mathbf{x}^{I}$ in $\left\{x_{1}, \ldots, x_{n}\right\}$, with $I=\left(m_{i}\right) \in \mathbb{Z}_{\geq 0}^{n}$, and $\gamma \in \Gamma$, such that $u:=\mathbf{x}^{I} \gamma$ occurs in the PBW expression for $g$ with non-zero coefficient, then $u$ would appear in $\sigma_{m}^{\mathcal{F}}\left(z_{1}\right)$, and could not be cancelled by any term from $\sigma_{m}^{\mathcal{F}}\left(z_{2}\right)$ since $\mathcal{M}$-degree $(u)=m$. This would contradict the fact that $\sigma_{m}^{\mathcal{F}}(z)=f$. Hence every basis term $u$ in $g$ has the form $\mathbf{x}^{I} \mathbf{y}^{J} \gamma$ with $J \neq(0, \ldots, 0)$. But $g \in \mathcal{M}_{m}(H)$, so that $|I|-|J|=m$, where $|I|=\sum_{i} m_{i}$. Therefore $|I|>m$, forcing $\mathcal{F}$-degree $(u)>m$. However this contradicts $z \in \mathcal{F}_{m} Z$, and so $g=0$. Therefore $z=z_{1}+z_{2}=f+z_{2}$. That is, $f=z_{1} \in \mathcal{M}_{m}(Z)$, so (12) is proved.

Since $\left(S(\mathfrak{h})^{\mathrm{p}}\right)^{\Gamma}$ is generated by homogeneous elements, it follows that $\left(S(\mathfrak{h})^{\mathrm{p}}\right)^{\Gamma} \subseteq Z$; and, by swapping the grading on $\mathfrak{h}$ and $\mathfrak{h}^{*}$, we can show in the same way that $\left(S\left(\mathfrak{h}^{*}\right)^{\mathrm{p}}\right)^{\Gamma} \subseteq Z$. Finally, the Poincaré-Birkhoff-Witt theorem implies that the subalgebra of $Z$ generated by these two invariant rings is $\left(S(\mathfrak{h})^{\mathrm{p}}\right)^{\Gamma} \otimes\left(S\left(\mathfrak{h}^{*}\right)^{\mathrm{p}}\right)^{\Gamma}$, completing the proof of the proposition.

Remark. Keep the notation of the proposition. By the Shepherd-Todd-Chevalley theorem, 2, Theorem 7.2.1], the central subalgebra $Z_{0}$ is a polynomial algebra in $2 n$ indeterminates. Moreover, by classical invariant theory $S(\mathfrak{h})$ [resp. $S\left(\mathfrak{h}^{*}\right)$ ] is a free $\left(S(\mathfrak{h})^{\mathrm{p}}\right)^{\Gamma}$-module [resp. $\left(S\left(\mathfrak{h}^{*}\right)^{\mathrm{p}}\right)^{\Gamma}$-module] of rank $p^{n}|\Gamma|$. Thus, by the Poincaré-Birkhoff-Witt theorem,

$$
H \text { is a free } Z_{0}-\text { module of rank } p^{2 n}|\Gamma|^{3} \text {. }
$$

Hence there is a bundle $\mathcal{B}$ of algebras of $k$-dimension $p^{2 n}|\Gamma|^{3}$ over affine $2 n$-space, and every irreducible $H$-module is a module for precisely one of the algebras in $\mathcal{B}$. Thus it makes sense to study the representation theory of $H$ by studying $\mathcal{B}$.

Example. Kleinian singularities of Type A. Let $r \in \mathbb{Z}, r>1$, with $r$ coprime to $p$, and let $\eta$ be a primitive $r$ th root of 1 in $k$. Let $\mathfrak{h}=k x, \mathfrak{h}^{*}=k y$, and let $\Gamma=\langle\gamma\rangle$ be the cyclic group of order $r$ acting on $\mathfrak{h}$ by $\gamma \cdot x=\eta x$, so that $\gamma \cdot y=\eta^{-1} y$. Thus, for $\mathbf{c}=\left(c_{1}, \ldots, c_{r-1}\right) \in k^{r}, H=H_{1, \mathbf{c}}\left(\mathfrak{h} \oplus \mathfrak{h}^{*}, \omega, \Gamma\right)$ is the algebra

$$
\begin{aligned}
k\langle x, y, \gamma: & \gamma x=\eta x \gamma, \gamma y=\eta^{-1} y \gamma \\
& {\left.[y, x]=1-\sum_{j=1}^{r-1} c_{j} \gamma^{j}\right\rangle . }
\end{aligned}
$$

One checks easily that

$$
x^{p r} \in Z(H), \quad y^{p r} \in Z(H) .
$$

It is convenient to have available also the basis of $k \Gamma$ afforded by the primitive idempotents $e_{j}:=\frac{1}{r} \sum_{i=0}^{r-1} \eta^{i j} \gamma^{i}$, for $j=0, \ldots, r-1$, with respect to which we 
write the commutator relation as

$$
[y, x]=\sum_{j=0}^{r-1} f_{j} e_{j}
$$

for $\mathbf{f}=\left(f_{j}\right) \in k^{n}$ with $\sum_{j} f_{j}=r$. The interested reader may write down the linear relations between the $c_{i}$ and the $f_{j}$. Define

$$
\tau=x y+\sum_{i=1}^{r-1}\left(i-\sum_{j=0}^{i-1} f_{j}\right) e_{i} \in H .
$$

Then $[\tau, x]=x$ and $[\tau, y]=-y$, so that

$$
h:=\tau^{p}-\tau \in Z(H) .
$$

Define elements $\left\{\delta_{m}: 0 \leq m \leq r-1\right\}$ of $k$ by

$$
\delta_{m}:=\sum_{j=1}^{r-1} c_{j}\left(1-\eta^{-j}\right)^{-1} \eta^{m j}=-\frac{1}{r} \sum_{l=0}^{r-1}\left(\rho_{m, l+1}\right) f_{l},
$$

where $\rho_{m, l+1}:=\sum_{j=1}^{r-1}\left[\eta^{(m+l) j} /\left(\eta^{j}-1\right)\right]$. The following result is proved by direct calculation in [8, Chapter 3].

Proposition. Let $H=H_{1, \mathbf{c}}$ be a symplectic reflection algebra for the Kleinian singularity of type A, as defined above. Keep the notation as above.

(i) $Z(H)=k\left\langle x^{p r}, y^{p r}, \tau\right\rangle$.

(ii) $Z(H) \cong k\left[X, Y, Z: X Y=\Pi_{m=0}^{r-1}\left(Z+\delta_{m}^{p}-\delta_{m}\right)\right]$.

(iii) $Z(H)$ is smooth if and only if $\delta_{i}-\delta_{j} \in \mathbb{Z}$ only when $i=j$.

4.3. The Dunkl embedding. One reason why rational Cherednik algebras over $\mathbb{C}$ at $t=1$ are easier to handle than arbitrary symplectic reflection algebras is that the $\mathbb{C}$-algebra $H_{\mathbb{C}}:=H_{1, \mathbf{c}}\left(\mathfrak{h} \oplus \mathfrak{h}^{*}, \omega, \Gamma\right)$ embeds in the skew group algebra $\mathcal{D}\left(\mathfrak{h}^{\text {reg }}\right) * \Gamma$; indeed $H_{\mathbb{C}}$ is birationally equivalent to the skew group algebra $\mathcal{D}(\mathfrak{h}) * \Gamma$ over the Weyl algebra $\mathcal{D}(\mathfrak{h})$, [12, Proposition 4.5].

In fact, the same is true in positive characteristic, with essentially the same proof. We keep the notation as in (4.1). In addition, for each pseudo-reflection $s \in S \subseteq \Gamma$ choose $\alpha_{s} \in \mathfrak{h}^{*}$ whose kernel is the hyperplane stabilized by $s$, and taking $\Gamma$-conjugates as appropriate so that $\delta:=\Pi_{s \in S} \alpha_{s} \in S\left(\mathfrak{h}^{*}\right)^{\Gamma}$. Write $\mathfrak{h}^{\text {reg }}$ for the regular points of $\mathfrak{h}$, so that $\mathcal{D}\left(\mathfrak{h}^{\text {reg }}\right)$, the algebra of differential operators on $\mathfrak{h}^{\text {reg }}$, is just $\mathcal{D}(\mathfrak{h})[\delta]^{-1}$, the localisation of the $n$th Weyl algebra $\mathcal{D}(\mathfrak{h})=$ $k\left[y_{1}, \ldots, y_{n}, \partial / \partial y_{1}, \ldots, \partial / \partial y_{n}\right]$ at the powers of $\delta$.

Theorem. Let $H$ be a rational Cherednik algebra, with notation as above. There are elements $\tau_{1}, \ldots, \tau_{n}$ of $k \Gamma$ such that the assignment $\gamma \mapsto \gamma, y_{i} \mapsto y_{i}$ and $x_{i} \mapsto$ $\partial / \partial y_{i}+\tau_{i}$ for $\gamma \in \Gamma$ and $i=1, \ldots, n$ extends to an injective algebra homomorphism $\Theta_{c}: H \longrightarrow \mathcal{D}\left(\mathfrak{h}^{\text {reg }}\right) * \Gamma$. 
Proof. The proof of [12, Proposition 4.5] works equally well in positive characteristic.

Remark: In fact, the proof yields a stronger statement: $\Theta_{\mathbf{c}}$ becomes an isomorphism after inverting $\delta$; that is, $H\left[\delta^{-1}\right] \cong \mathcal{D}\left(\mathfrak{h}^{\text {reg }}\right) * \Gamma$.

4.4. Goldie rank. Recall [22, Theorem 10.4.4] that the Goldie or uniform rank $\operatorname{udim}_{R}(M)$ of a module $M$ over the noetherian ring $R$ is the biggest number of nonzero modules whose direct sum embeds into $M$, or infinity if no such supremum exists. If $\mathcal{C}$ is an Ore set of non-zero-divisors in $R$ and $M$ is $\mathcal{C}$-torsion free then it is easy to check that

$$
\operatorname{udim}_{R}(M)=\operatorname{udim}_{R \mathcal{C}^{-1}}\left(M \otimes_{R} R \mathcal{C}^{-1}\right),
$$

[22, proof of Lemma 10.2.13]. Apply this in particular when $R$ is a prime noetherian ring, $\mathcal{C}$ is the set of all non-zero-divisors in $R$, and $M=R$. In this case $\mathcal{C}$ is an Ore set and $R C^{-1}$ is the simple artinian quotient ring $Q(R)$ of $R$, by Goldie's theorem, [22, Theorem 10.4.10]. Thus $Q(R) \cong M_{s}(D)$ for a division ring $D$ by the Artin-Wedderburn theorem, and (15) shows that the integer $s$ is the Goldie rank of $R$.

Theorem. Let $H=H_{1, \mathbf{c}}(\mathfrak{h}, \omega, \Gamma)$ be a rational Cherednik algebra.

(i) $\operatorname{udim}(H)=|\Gamma|$.

(ii) The integer s appearing in Theorem 3.4 is the Goldie rank of $H$, and so equals $|\Gamma|$.

Proof. Fix a prime $\mathfrak{p}$ of $Z(H)$ which is in the Azumaya locus. Then the isomorphism of Theorem 3.4(ii) holds, and since $e H_{\mathfrak{p}} e$ is a domain by Theorem (3.1) (ii), the first claim in (ii) follows. Thus it remains to prove that $\operatorname{udim}(H)=|\Gamma|$. Since the Goldie rank of an algebra is unaltered by inverting an Ore set of non-zero-divisors, by (15), in view of Theorem (4.3) we only need to show that

$$
\operatorname{udim}(\mathcal{D}(\mathfrak{h}) * \Gamma)=|\Gamma|
$$

This follows from a special case of Moody's theorem, [24, Theorem 37.14].

Remark: Presumably Theorem 4.4(i) is true for all symplectic reflection algebras over all fields. Suppose first that $K$ is algebraically closed of characteristic 0 . Then the Dunkl embedding can be used as above to deal with rational Cherednik $K$-algebras when $t=1$. Secondly, if $K$ has any characteristic and $H$ is any symplectic reflection algebra with $t=0$, then $e H e$ is commutative by [12, Theorem 3.1], so the integer $s$ of Theorem 3.4(ii) is equal to the PI-degree of $H$. But the latter is equal to the dimension over the algebraically closed field $k$ of a generic irreducible representation of $H$, by [7, Theorem III.1.6 and Lemma III.1.2], and this is known to be $|\Gamma|$ by [12, Theorem 1.7(iv)]. 
Thus the only case remaining open is that of a symplectic reflection algebra which is not Cherednik, with $t=1$.

\section{Cherednik algebras - Representation theory}

5.1. PI-degree and centre. When $k$ has characteristic 0 and $t=0$, the PI-degree of a symplectic reflection algebra $H_{0, \mathbf{c}}$ is $|\Gamma|$, [12, Theorem 1.7(iv)]; indeed the irreducible $H_{0, \mathbf{c}}$-modules in the Azumaya locus are isomorphic as $k \Gamma$-modules to $k \Gamma$. The same conclusions remain valid when $t=0$ and $k$ has positive characteristic, with essentially the same proofs. It remains to consider the case $t=1$ when $k$ has positive characteristic; here we deal with the Cherednik algebras. For this we need the following two well-known facts:

Lemma. (i) The centre of the Weyl algebra $\mathcal{D}(\mathfrak{h})=k\left\langle x_{1}, \ldots, x_{n}, y_{1}, \ldots, y_{n}\right\rangle$ is $k\left\langle x_{1}^{p}, \ldots, x_{n}^{p}, y_{1}^{p}, \ldots, y_{n}^{p}\right\rangle$; indeed $\mathcal{D}(\mathfrak{h})$ is Azumaya over $k\left\langle x_{1}^{p}, \ldots, x_{n}^{p}, y_{1}^{p}, \ldots, y_{n}^{p}\right\rangle$.

(ii) Let $R$ be a domain and let $G$ be a finite group of automorphisms of $R$ which acts faithfully on $Z(R)$. Then the centre of the skew group ring $R * G$ is $Z(R)^{G}$.

Proof. (i) is proved in [25] and (ii) is a straightforward exercise.

Theorem. Let $H=H_{1, \mathbf{c}}\left(\mathfrak{h} \oplus \mathfrak{h}^{*}, \omega, \Gamma\right)$ be a rational Cherednik algebra over an algebraically closed field $k$ of positive characteristic $p$. Let $\mathfrak{h}$ have dimension $n$. Then

$$
\mathrm{PI}-\operatorname{degree}(H)=p^{n}|\Gamma| .
$$

Proof. By Theorem and Remark 4.3, $H$ and $\mathcal{D}(\mathfrak{h}) * \Gamma$ become isomorphic after inverting certain central elements, so it is enough, by [22, Lemma 10.2.13], to prove that $\mathcal{D}(\mathfrak{h}) * \Gamma$ has PI-degree $p^{n}|\Gamma|$. From the lemma we deduce that

$$
Z:=Z(\mathcal{D}(\mathfrak{h}) * \Gamma) \quad=\quad k\left\langle x_{1}^{p}, \ldots, x_{n}^{p}, y_{1}^{p}, \ldots, y_{n}^{p}\right\rangle^{\Gamma} .
$$

Now, inverting the non-zero elements of the centre in $\mathcal{D}(\mathfrak{h}) * \Gamma$ and calculating that

$$
\operatorname{dim}_{Q(Z)}\left(Q(Z) \otimes_{Z} \mathcal{D}(\mathfrak{h}) * \Gamma\right) \quad=\quad p^{2 n}|\Gamma|^{2},
$$

we obtain the desired conclusion.

Presumably Theorem 5.1 is true for all symplectic reflection algebras with $t=1$ over a characteristic $p$ field; we leave this as an open question.

Corollary. Let $H=H_{1, \mathbf{c}}\left(\mathfrak{h} \oplus \mathfrak{h}^{*}, \omega, \Gamma\right)$ be a rational Cherednik algebra over an algebraically closed field $k$ of positive characteristic $p$. Then $Z(H)$ is a free module of rank $|\Gamma|$ over its polynomial subalgebra $Z_{0}=\left(S\left(\mathfrak{h}^{p}\right)^{\Gamma} \otimes\left(S\left(\mathfrak{h}^{* p}\right)^{\Gamma}\right.\right.$. 
Proof. Let $\mathfrak{h}$ have dimension $n$. By Theorem 2.3(iv), $Z(H)$ is Gorenstein, and therefore Cohen-Macaulay. Thus, by [11, Corollary 18.17], it is free over its polynomial subalgebra $Z_{0}$. The rank is determined by comparing $\operatorname{dim}_{Q\left(Z_{0}\right)}\left(Q\left(Z_{0}\right) \otimes H\right)$ with $\operatorname{dim}_{Q(Z)}\left(Q\left(Z_{0}\right) \otimes H\right)$; the first of these is $p^{2 n}|\Gamma|^{3}$ by (18), while the second is $p^{2 n}|\Gamma|^{2}$ by Theorem 5.1 .

5.2. $\Gamma$-regularity of the generic irreducible modules. It follows from Theorem 5.1 that the maximal $k$-dimension of the irreducible $H$-modules is $p^{n}|\Gamma|$. By the structure theory of noetherian PI-rings, this dimension is achieved precisely by those irreducible $H$-modules $V$ for which $\mathfrak{m}:=\operatorname{Ann}_{Z(H)}(V)$ has the property that $H / \mathfrak{m} H$ is simple artinian, and in this case $H / \mathfrak{m} H \cong M_{p^{n}|\Gamma|}(k)$. The open set of such $\mathfrak{m}$ is precisely the Azumaya locus of (3.4). Recall that [12, Theorem 1.7(vi)] shows that, for any symplectic reflection algebra over any field, at $t=0$, the irreducible modules of maximal dimension are $k \Gamma$-regular of rank one. Analogously, we can describe the $k \Gamma$-structure of the Azumaya irreducibles over Cherednik algebras for $t \neq 0$ in positive characteristic. We begin with an easy lemma which essentially ensures that the desired result is true for $H_{1, \mathbf{0}}$.

Lemma. Let $k$ have characteristic $p>0$ as usual, and let $(V, \omega)$ be a symplectic $k$-vector space with basis $\left\{x_{1}, \ldots, x_{n}, y_{1}, \ldots, y_{n}\right\}$, with $\omega\left(x_{i}, y_{j}\right)=\delta_{i j}, \omega\left(x_{i}, x_{j}\right)=$ $\omega\left(y_{i}, y_{j}\right)=0$. Let $\Gamma$ be a finite subgroup of $\operatorname{Sp}(V)$, of order prime to $p$. Write $\mathfrak{h}$ for the subspace $\sum_{i} k x_{i}$ of $V$, so that $\Gamma$ acts by automorphisms on both $S(V)$ and $\mathcal{D}(\mathfrak{h})$. Let $F$ be the quotient field of $\left(S(V)^{\Gamma}\right)^{\mathrm{p}}$. Then $F \otimes_{\left(S(V)^{\Gamma}\right)_{\mathrm{p}}^{\mathrm{p}}} \mathcal{D}(V)$ is a free $F \Gamma$-module of rank $p^{m}$.

Proof. Note first that $\left(S(V)^{\Gamma}\right)^{\mathrm{p}}$ is a central $\Gamma$-invariant subalgebra of $\mathcal{D}(\mathfrak{h})$, so the statement of the lemma makes sense. Moreover, as $F \Gamma$-modules, there is no difference between $F \otimes_{\left(S(V)^{\Gamma}\right)^{\text {p }}} \mathcal{D}(\mathfrak{h})$ and $F \otimes_{\left(S(V)^{\Gamma}\right)^{\text {p }}} S(V)$, so we can work with the latter. Then $F \otimes S(V)$ is simply the quotient field of $S(V)$, which is a free $Q\left(S(V)^{\Gamma}\right) \Gamma$-module of rank one by the Primitive Element Theorem of Galois theory. Since $\operatorname{dim}_{F}\left(Q\left(S(V)^{\Gamma}\right)\right)=p^{m}$, the result follows.

Theorem. Let $H$ be as in Theorem [5.1, and let the maximal ideal $\mathfrak{m}$ of $Z=Z(H)$ be in the Azumaya locus of $H$.

(i) $\mathrm{He} / \mathfrak{m} H e$ is a regular $k \Gamma$-module of rank $p^{2 n}$.

(ii) The irreducible $H$ - module $V$ with $\operatorname{Ann}_{Z}(V)=\mathfrak{m}$ is a regular $k \Gamma$-module of rank $p^{n}$.

Proof. (i) By Theorems 3.4(ii) and 4.4(ii), for every prime $\mathfrak{p}$ of $Z$ in the Azumaya locus of $H$,

$$
H_{\mathfrak{p}} \cong M_{|\Gamma|}\left(e H_{\mathfrak{p}} e\right) .
$$

Set $Q:=Q(Z)$, the quotient field of $Z$, so by Theorem [5.1 we have

$$
\operatorname{dim}_{Q}\left(Q \otimes_{Z} H\right)=p^{2 n}|\Gamma|^{2} .
$$


Thus (16) implies that

$$
\operatorname{dim}_{Q}\left(Q \otimes_{Z} H e\right)=p^{2 n}|\Gamma| .
$$

Now let $\mathfrak{m}$ be a maximal ideal of $Z$ in the Azumaya locus of $H$. The Azumaya property ensures that $H_{\mathfrak{m}}$, and hence also $H_{\mathfrak{m}} e$, are projective and thus free $Z_{\mathfrak{m}}$-modules. In particular, from (17),

$$
H_{\mathfrak{m}} \text { e is } Z_{\mathfrak{m}-} \text {-free of rank } p^{2 n}|\Gamma| .
$$

Now let $\operatorname{Irr}(k \Gamma)$ be the set of isomorphism classes of irreducible $k \Gamma$-modules. We decompose $\mathrm{He}$ as the direct sum of its isotypic components as left $k \Gamma$-module:

$$
H e=\bigoplus_{E \in \operatorname{Irr}(k \Gamma)} \operatorname{Isot}_{E}(H e)
$$

Of course this is a sum of $Z$-modules as well as $k \Gamma$-modules, so applying $Q \otimes_{Z}-$ to (19) yields

$$
Q \otimes_{Z} H e=\bigoplus_{E \in \operatorname{Irr}(k \Gamma)}\left(Q \otimes_{Z} \operatorname{Isot}_{E}(H e)\right) .
$$

Thanks to the Dunkl embedding, Theorem and Remark 4.3, $H$ is birationally equivalent to the skew group algebra $\mathcal{D}(\mathfrak{h}) * \Gamma$, via a map which is the identity when restricted to $k \Gamma$. By this and the above lemma,

$$
Q \otimes_{Z} H e \text { is } Q \Gamma \text {-regular of rank } p^{2 n} .
$$

By (18), the localised isotypic components

$$
Z_{\mathfrak{m}} \otimes_{Z} \operatorname{Isot}_{E}(H e) \cong \operatorname{Isot}_{E}\left(H_{\mathfrak{m}} e\right)
$$

are $Z_{\mathfrak{m}}$-free for each $k \Gamma$-irreducible $E$; and so, in view of (20),

$$
Z_{\mathfrak{m}} \otimes_{Z} \operatorname{Isot}_{E}(H e) \text { has } Z_{\mathfrak{m}}-\operatorname{rank} p^{2 n}\left(\operatorname{dim}_{k}(E)\right)^{2} .
$$

We deduce from this that, factoring $Z_{\mathfrak{m}}$ and the isotypic component by $\mathfrak{m} Z_{\mathfrak{m}}$,

$$
\operatorname{dim}_{k}\left(\operatorname{Isot}_{E}(H e / \mathfrak{m} H e)=p^{2 n}\left(\operatorname{dim}_{k}(E)\right)^{2} .\right.
$$

That is, the multiplicity of $E$ in $H e / \mathfrak{m} H e$ is $p^{2 n} \operatorname{dim}_{k}(E)$, proving (i).

(ii) By Theorem 5.1, $\operatorname{dim}_{k}(V)=p^{n}|\Gamma|$. Since $V$ is the unique irreducible module for the simple artinian ring $\mathrm{H} / \mathfrak{m} H, H e / \mathfrak{m} H e$ is the sum of $p^{n}$ copies of $V$. Therefore it follows from (i) that $V$ is $k \Gamma$-regular of rank $p^{n}$.

\subsection{Azumaya versus smooth locus.}

Theorem. Let $H=H_{1, \mathbf{c}}(\mathfrak{h}, \omega, \Gamma)$ be a rational Cherednik algebra over an algebraically closed field $k$ of positive characteristic p. Let $Z$ be the centre of $H$, and write $\mathcal{A}_{H}$ for the Azumaya locus of $Z$ in $H$, and $\mathcal{S}_{Z}$ for the singular locus of $\operatorname{Maxspec}(Z)$. Then

$$
\mathcal{A}_{H}=\operatorname{Maxspec}(Z) \backslash \mathcal{S}_{Z}
$$


Proof. Since $H$ is a finite $Z$-module and is Auslander-regular and Cohen-Macaulay, by Theorems 2.3(ii) and 2.2(iii), it follows from [6, Theorem 3.8] that it's enough to prove that $H$ is Azumaya in codimension one. That is, let $\mathfrak{p}$ be a prime ideal of $Z$ of height one. We must show that $H_{\mathfrak{p}}$ is Azumaya; equivalently we must exhibit a maximal ideal $\mathfrak{m}$ of $Z$ with $\mathfrak{m}$ Azumaya and $\mathfrak{p} \subseteq \mathfrak{m}$. Let $Z_{0}$ be the polynomial subalgebra $\left(S(\mathfrak{h})^{\mathrm{p}}\right)^{\Gamma} \otimes\left(S\left(\mathfrak{h}^{*}\right)^{\mathrm{p}}\right)^{\Gamma}$ of $Z$ provided by Proposition 4.2. Thus $\mathfrak{p}_{0}:=\mathfrak{p} \cap Z_{0}$ is a prime ideal of $Z_{0}$, and

$$
\operatorname{height}\left(\mathfrak{p}_{0}\right)=1 \text {, }
$$

by Lying Over, [11, Proposition 4.15]. We claim that

$$
\text { either } \mathfrak{p}_{0} \cap\left(S(\mathfrak{h})^{\mathrm{p}}\right)^{\Gamma}=0 \text { or } \mathfrak{p}_{0} \cap\left(S\left(\mathfrak{h}^{*}\right)^{\mathrm{p}}\right)^{\Gamma}=0 .
$$

For, suppose for a contradiction that both intersections are non-zero. Then

$$
\mathfrak{q}:=\left(\mathfrak{p}_{0} \cap\left(S(\mathfrak{h})^{\mathrm{p}}\right)^{\Gamma}\right) Z_{0}=\left(\mathfrak{p}_{0} \cap\left(S(\mathfrak{h})^{\mathrm{p}}\right)^{\Gamma}\right) \otimes\left(S\left(\mathfrak{h}^{*}\right)^{\mathrm{p}}\right)^{\Gamma}
$$

is a non-zero prime of $Z_{0}$ contained in $\mathfrak{p}_{0}$, and clearly $\mathfrak{q} \cap\left(S\left(\mathfrak{h}^{*}\right)^{\mathrm{p}}\right)^{\Gamma}=0$, so that $\mathfrak{q} \varsubsetneqq \mathfrak{p}_{0}$. But this contradicts (21), and hence (22) is true.

Let's suppose first that $\mathfrak{p}_{0} \cap\left(S\left(\mathfrak{h}^{*}\right)^{\mathrm{p}}\right)^{\Gamma}=0$. Then, in particular, $\mathfrak{p}$ does not contain the element $\delta$ defined in (4.3), and hence there is a maximal ideal $\mathfrak{m}$ of $Z$ with $\mathfrak{p} \subseteq \mathfrak{m}$, such that $\mathfrak{m}$ does not contain $\delta$. We claim that $\mathfrak{m}$ is Azumaya; in view of [5, Theorem III.1.6] and Theorem [5.1, this amounts to showing that, if $W$ denotes an irreducible $H$-module killed by $\mathfrak{m}$, then

$$
\operatorname{dim}_{k}(W)=p^{n}|\Gamma|
$$

Now $\delta$ acts as multiplication by a non-zero scalar on $W$; so, since $H[\delta]^{-1} \cong$ $\mathcal{D}\left(\mathfrak{h}^{\text {reg }}\right) * \Gamma$ by Theorem and Remark 4.3, $W$ admits actions of (i) $\mathcal{D}\left(\mathfrak{h}^{\text {reg }}\right)$, and of (ii) $S\left(\mathfrak{h}^{*}\right)[\delta]^{-1} * \Gamma$.

From (i) and Lemma $5.1($ i) we deduce that

$$
p^{n} \mid \operatorname{dim}_{k}(W) .
$$

Let $U$ be any irreducible $S\left(\mathfrak{h}^{*}\right)[\delta]^{-1} * \Gamma$-module, so $\operatorname{dim}_{k}(U)<\infty$ and so there is a maximal ideal $\mathfrak{t}$ of $S\left(\mathfrak{h}^{*}\right)[\delta]^{-1}$ and $0 \neq u \in U$ with $\mathfrak{t} u=0$. Set $U_{1}:=\operatorname{Ann}_{U}(\mathfrak{t})$, a nonzero $S\left(\mathfrak{h}^{*}\right)[\delta]^{-1}$-submodule of $U$. For each $\gamma \in \Gamma, \gamma U_{1}=\operatorname{Ann}_{U}\left(\mathfrak{t}^{\gamma}\right)$ is isomorphic as a vector space to $U_{1}$. Now $\mathfrak{t}$ has $|\Gamma|$ distinct $\Gamma$-conjugates, by definition of $\delta$. Consider $U^{\prime}:=\sum_{\gamma \in \Gamma} \gamma U_{1} \subseteq U$. Clearly $U^{\prime}$ is a non-zero $S\left(\mathfrak{h}^{*}\right)[\delta]^{-1} * \Gamma$-submodule of $U$, and therefore $U^{\prime}=U$. Moreover the sum in the definition of $U^{\prime}$ has $|\Gamma|$ distinct terms, each term killed by a distinct maximal ideal. So the sum is direct, and hence $\operatorname{dim}_{k}(U)=|\Gamma| \operatorname{dim}_{k}\left(U_{1}\right)$. In particular, $|\Gamma| \mid \operatorname{dim}_{k}(U)$; since $W$ has a finite composition series as $S\left(\mathfrak{h}^{*}\right)[\delta]^{-1} * \Gamma$-module,

$$
|\Gamma| \mid \operatorname{dim}_{k}(W) .
$$


Combining (24) and (25), recalling that $p \nmid|\Gamma|$ by hypothesis, proves (23), and so the theorem follows.

\section{Questions And CONJECtures}

Throughout, $H=H_{1, \mathbf{c}}(V, \omega, \Gamma)$ is a symplectic reflection algebra over $k$, which is algebraically closed of characteristic $p>0$. Let $V$ have dimension $2 n$.

We repeat the question about Goldie ranks which was stated, with background discussion, in Remark 4.4.

Question A: Does $H$ have Goldie dimension $|\Gamma|$ :1

Similarly, it seems reasonable to expect that the value for the PI-degree of Cherednik algebras obtained in (5.1) applies in general:

Question B: Does $H$ have PI-degree $p^{n}|\Gamma|$ ?

A more precise version of the above question is:

Question C: Is every simple $H$-module of maximal dimension a regular $k \Gamma$-module of rank $p^{n}$ ?

It is of interest from the perspective of noncommutative resolutions of singularities to ask:

Question D: For which $H$ do there exist values of the parameter $\mathbf{c}$ for which $\operatorname{Maxspec}(Z(H))$ is smooth? When such values exist determine them all.

The analogue of the first part of Question D in characteristic 0 at $t=0$ has been answered completely, as a result of a considerable body of work - see [12, 14, 20, 1,. A natural strategy to attack this problem in the Cherednik case is afforded by Theorem 5.3. To have this route available in the setting of an arbitrary symplectic reflection algebra, one needs therefore to answer:

Question E: Does the Azumaya locus coincide with the smooth locus for an arbitrary symplectic reflection algebra?

Work on the finite dimensional representation theory in characteristic 0 is considerably helped by the underlying Poisson structure - in view of [7, Theorems 4.2 and 7.8], there are only finitely many symplectic leaves in $\operatorname{Maxspec}(Z(H))$, and the representation theory is constant across leaves, in the sense that, if $\mathfrak{m}$ and $\mathfrak{n}$ belong to the same leaf, then $H / \mathfrak{m} H \cong H / \mathfrak{n} H$. This motivates:

Question F: Are there only finitely many isomorphism classes of factors $H / \mathfrak{m} H$ as $\mathfrak{m}$ ranges through $\operatorname{Maxspec}(Z(H))$ ?

The annoying gap in the equivalences of Theorem 3.4 is one indication that the symmetrising subalgebra is not very well understood. We therefore ask:

Question G: Is every localizable prime ideal $P$ of $H$ generated by its intersection with $Z(H)$ ?

\footnotetext{
${ }^{1}$ We understand that Iain Gordon has been able to confirm this.
} 


\section{REFERENCES}

[1] G. Bellamy, On singular Calegoro-Moser spaces, arXiv math RT/0707.3694

[2] D.J. Benson, Polynomial Invariants of Finite Groups, London Math. Soc. Lecture Notes 190, Cambridge, 1993.

[3] R. Bezrukavnikov, M. Finkelberg and V. Ginzburg, with an Appendix by P. Etingof, Cherednik algebras and Hilbert schemes in characteristic $p$, Represent. Theory 10 (2006), 254298.

[4] J.-E. Bjork, The Auslander condition in Noetherian rings, Séminaire d'Algèbre Paul Dubreil et Marie-Paule Malliavin, Lecture Notes in Math., 1404, 137-173, Springer, (Berlin), 1989.

[5] K.A. Brown and K.R. Goodearl, Lectures on Algebraic Quantum Groups, Advanced Courses in Mathematics, CRM Barcelona, Birkhäuser Verlag, Basel, 2002.

[6] K.A. Brown and K.R. Goodearl, Homological aspects of noetherian PI Hopf algebras and irreducible modules of maximal dimension, J. Algebra 198 (1997), 240-265.

[7] K.A. Brown and I. Gordon, Poisson orders, symplectic reflection algebras and representation theory, J. reine angew. Math. 559 (2003), 193-216.

[8] K. Changtong, Symplectic Reflection Algebras in Positive Characteristic, Ph.D. thesis, University of Glasgow, August 2006.

[9] S.C. Coutinho, A Primer of Algebraic D-modules, London Math. Soc. Student Texts 33, CUP, 1995.

[10] W. Crawley-Boevey and M.P. Holland, Noncommutative deformations of Kleinian singularities, Duke Math. J. 92 (1998), 605-635.

[11] D. Eisenbud, Commutative Algebra with a view towards Algebraic Geometry Springer Verlag (Berlin), 1995.

[12] P. Etingof and V. Ginzburg, Symplectic reflection algebras, Calogero-Moser space, and deformed Harish-Chandra homomorphism, Invent.Math. 147 (2002), 243-348.

[13] K. R. Goodearl and R. W. Warfield Jr., An Introduction to Noncommutative Noetherian Rings, Second edition, London Mathematical Society Student Texts, 61, Cambridge University Press, Cambridge, 2004.

[14] I. Gordon, Baby Verma modules for rational Cherednik algebras, Bulli London Math. Soc. 35 (2003), 321-336.

[15] S. Koenig, I.H. Slungard and C. Xi, Double centralizer properties, dominant dimension, and tilting modules, J. Algebra 240 (2001), 393-412.

[16] G. Krause and T.H. Lenagan, Growth of Algebras and Gel'fand-Kirillov Dimension, Graduate Studies in Mathematics vol. 22, Amer. Math. Soc., Providence, R.I., 2000.

[17] F. Latour, Representations of rational Cherednik algebras of rank 1 in positive characteristic, J. Pure Appl. Algebra 195 (2005), 97112.

[18] Li Huishi and F. Van Oystaeyen, Zariskian Filtrations, Kluwer, Dordrecht, 1996.

[19] R. Martin, Skew group rings and maximal orders, Glasgow Math. J. 37 (1995), 249-263.

[20] M. Martino, Stratifications of Marsden-Weinstein reductions for representations of quivers and deformations of symplectic quotient singularities, arXiv math/0603562

[21] G. Maury and J. Raynaud, Ordres Maximaux au sens d'Asano, Springer Lecture Notes in Math., vol 808, Springer (Berlin), 1980.

[22] J.C. McConnell and J.C. Robson, Noncommutative Noetherian Rings, Academic Press (New York), 1987.

[23] B.J.Müller, Localisation in non-commutative Noetherian rings, Can. J. Math. 28 (1976), 600-610.

[24] D.S. Passman, Infinite Crossed Products, Academic Press (New York) 1989.

[25] P. Revoy, Algèbres de Weyl en characteristiques p, C.R. Acad. Sci. Paris (A), 276 (1973), 225-228. 
[26] K. Watanabe, Certain invariant rings are Gorenstein, I, II, Osaka J. Math., 11(1974), 1-8 and 379-388.

[27] Z. Yi, Injective homogeneity and the Auslander-Gorenstein property, Glasgow Math. J. 37 (1995), 191-204.

[28] A. Zaks, Injective dimension of semiprimary rings, J. Algebra 13 (1969), 73-86.

Brown: Department of Mathematics, University of Glasgow, Glasgow G12 8QW, UK

E-mail address: kab@maths.gla.ac.uk

Changtong: Department of Mathematics, Faculty of Science, Ubon Ratchathani UniVERsity, Ubon Ratchathani, 34190, Thailand.

E-mail address: noikanok@gmail.com 